Technological University Dublin

DÜBLIN

ARROW@TU Dublin

Articles

Crest: Centre for Research in Engineering

Surface Technology

2007

\title{
Silver Doped Titanium Dioxide Nanomaterials for Enhanced \\ Visible Light Photocatalysis
}

\author{
Michael Seery \\ Technological University Dublin, michael.seery@tudublin.ie \\ Reena George \\ Suresh Pillai \\ Technological University Dublin, suresh.pillai@tudublin.ie
}

See next page for additional authors

Follow this and additional works at: https://arrow.tudublin.ie/cenresart

Part of the Inorganic Chemistry Commons

\section{Recommended Citation}

Seery, M.K. et al. (2007) Silver doped titanium dioxide nanomaterials for enhanced visible light photocatalysis, Journal of Photochemistry and Photobiology A, vol. 189, no. 2-3, 2007, 258-263. doi:10.1016/j.jphotochem.2007.02.010

This Article is brought to you for free and open access by the Crest: Centre for Research in Engineering Surface Technology at ARROW@TU Dublin. It has been accepted for inclusion in Articles by an authorized administrator of ARROW@TU Dublin. For more information, please contact arrow.admin@tudublin.ie, aisling.coyne@tudublin.ie,gerard.connolly@tudublin.ie.

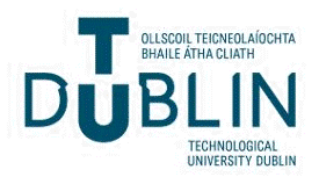


Authors

Michael Seery, Reena George, Suresh Pillai, and Patrick Floris

This article is available at ARROW@TU Dublin: https://arrow.tudublin.ie/cenresart/45 


\title{
Silver doped titanium dioxide nanomaterials for enhanced visible light photocatalysis
}

\author{
Michael K. Seery ${ }^{a}{ }^{*}$, Reenamole George ${ }^{a}$, Patrick Floris ${ }^{a}$, Suresh C. Pillai ${ }^{b}$ \\ ${ }^{a}$ School of Chemical and Pharmaceutical Sciences, Dublin Institute of Technology, Kevin St., Dublin 8, Ireland \\ ${ }^{\mathrm{b}}$ Centre for Research in Engineering Surface Technology (CREST), Dublin Institute of Technology, Camden Row, Dublin 8, Ireland
}

Received 4 December 2006; received in revised form 30 January 2007; accepted 14 February 2007

visible light activity to $\mathrm{TiO}_{2}[5-10]$ and these studies include the doping with metal and non-metal ions [11-14], dye sensitisation [15], and semiconductor coupling, etc.

As well as the energy of incident light, the photocatalytic efficiency depends on the rate of recombination of photoexcited electrons and holes. The photocatalytic mechanism involves the excitation of valence electrons to the conduction band by absorbing the UV light, resulting in the formation of holes in the valence band [16]. These electrons and holes can undergo subsequent reduction and oxidation before the recombination reaction. However, the lifetimes of these species are very small and in the absence of suitable scavengers, they will dissipate the stored energy within a few nanoseconds by recombination. Many studies have been carried out to improve the photocatalytic activity by reducing the recombination reaction by the insertion of noble metals [17-19] and it is found that doping with silver has been of considerable interest because of its potential applications. The importance in medical applications of silver and antibacterial activity of $\mathrm{TiO}_{2}$ together led researchers to think about the manufacture of silver doped titania coated sanitary wares [20], medical devices, food preparation surfaces, air conditioning filters, etc. Silver can trap the excited electrons from 
$\mathrm{TiO}_{2}$ and leave the holes for the degradation reaction of organic species $[21,22]$. It also results in the extension of their wavelength response towards the visible region [23-25]. Moreover, silver particles can facilitate the electron excitation by creating a local electric field [26], and plasmon resonance effect in metallic silver particles shows a reasonable enhancement in this electric field [15]. The effect of Ag doping on titania and its photocatalytic activity by UV irradiation was studied by Chao et al. [11] (by the sol-gel method) and they found that Ag doping promotes the anatase to rutile transformation, which is attributed to the increase in specific surface area which causes the improvement in photocatalytic activity, and enhances the electron-hole pair separation.

There are some contradictory results also reported showing the decreased activity of silver modified titania $[28,29]$. This may be due to their preparation method, nature of organic molecules, photoreaction medium, or the metal content and its dispersion. Even though there are many studies showing the photocatalytic activity of silver doped titania [11,31-33] the exact mechanism and the role of silver is under debate.

In this paper we report a systematic comparative study of solar initiated photocatalytic activity of sol-gel titania with silver nitrate added in various amounts. The silver ion was either reduced during the synthesis phase by irradiating the sample with light (pre-irradiated) or formed during calcination of the sol-gel material via decomposition of silver nitrate (not preirradiated). This method allows for the formation of sol-gel derived, homogeneously silver doped stable titania. We examined the structural characteristics of this material using X-ray diffraction (XRD), electron microscopy and absorption spectra and the effect of these materials on the photodegradation of a model dye, rhodamine 6G (R6G).

\section{Experimental}

Titanium tetraisopropoxide (97\%), acetic acid (99.7\%) and silver nitrate (99\%) were purchased from Aldrich. Rhodamine $6 \mathrm{G}$ was purchased from Eastman and was of analytical reagent grade and used without further purification. Deionised water was used in all experimental preparations. The samples were prepared by a modified sol-gel route [34]. Titanium isopropoxide $(25 \mathrm{ml})$ was added to acetic acid $(48 \mathrm{ml})$ with stirring. Then water $(150 \mathrm{ml})$ was added to the mixture dropwise with vigorous stirring. (The titanium isopropoxide, acetic acid and water are in 1:10:100 molar ratios.) The solution was stirred for $8 \mathrm{~h}$ to get a clear transparent sol and allowed to dry at $100^{\circ} \mathrm{C}$, after which it was calcined at $600^{\circ} \mathrm{C}$ in air for $2 \mathrm{~h}$ at a ramp rate of $5^{\circ} \mathrm{C} / \mathrm{min}$. To prepare silver doped titania, the above procedure was repeated, including silver nitrate $(1,2,3$ and $5 \mathrm{~mol} \%)$ when adding water to the titanium isopropoxide mixture. The sol was then either irradiated with $250 \mathrm{~W}$ bulb for $60 \mathrm{~min}$ ("pre-irradiated samples") after which they were kept in an oven set at $100^{\circ} \mathrm{C}$ until all the solvent evaporated or directly placed in the oven ("not preirradiated"). The dried powders were calcined at $600^{\circ} \mathrm{C}$ for $2 \mathrm{~h}$ at a ramp rate of $5{ }^{\circ} \mathrm{C} / \mathrm{min}$. The photocatalytic activities of the materials were studied by examining the degradation reaction of R6G. For powder samples, about $0.06 \mathrm{~g}$ of titania sample was dispersed in $50 \mathrm{ml}$ of R6G solution having concentration $5 \times 10^{-6} \mathrm{M}$. This suspension was irradiated with light using QSun solar test chamber $\left(0.68 \mathrm{~W} / \mathrm{m}^{2}\right.$ at $\left.340 \mathrm{~nm}\right)$. The suspension was stirred during irradiation, but was not purged with any gas. Degradation was monitored by collecting aliquots at increasing time intervals. The aliquots were centrifuged and absorption spectra were recorded using Perkin-Elmer Lambda 900 spectrophotometer. The terrestrial activity of the highly active silver doped sample and the standard $\mathrm{TiO}_{2}$ was determined by carrying out the reaction under Dublin summer sunlight at noon. The intensity of the sunlight was determined by a Solar light Co. Broadband radiometer PMA 2107 (Philadelphia) [35] which gave approximately $12.4 \mathrm{~W} / \mathrm{m}^{2}$ summed across the $260-400 \mathrm{~nm}$ wave band. Band gaps of the coated materials were measured by recording the transmission spectra of spin-coated films on glass. XRD were collected using a Siemens D $500 \mathrm{X}$-ray diffractometer, with a diffraction angle range $2 \theta=10-70^{\circ}$ using $\mathrm{Cu}$ $\mathrm{K} \alpha$ radiation. UV-vis absorption spectra were obtained on a Perkin-Elmer Lambda 900 spectrophotometer.

The mass fraction of rutile in the calcined samples was calculated by Spurr formula (Eq. (1)) which is the relationship between integrated intensities of anatase ( $\left.\begin{array}{llll}1 & 0 & 1\end{array}\right)$ and rutile (llll 110$)$ peaks where $I_{\mathrm{A}}$ and $I_{\mathrm{R}}$ are the integrated peak intensities of anatase and rutile peaks, respectively.

$$
X_{\mathrm{R}}=\frac{1}{1+0.8\left(I_{\mathrm{A}} / I_{\mathrm{R}}\right)}
$$

Particle size was determined by the Scherrer equation which calculates crystalline size using the expression $(0.9 \lambda /(\beta \cos \theta))$, where $\lambda$ is the $\mathrm{X}$-ray wavelength, $\theta$ the Bragg angle and $\beta$ is the line broadening.

\section{Results}

\subsection{XRD analysis}

XRD of the doped and undoped titania samples irradiated with light for $40 \mathrm{~min}$ to reduce the silver nitrate and calcined at $600^{\circ} \mathrm{C}$ are shown in Fig. 1. The materials show a high degree of crystallinity and existence of fully anatase phase at $600^{\circ} \mathrm{C}$. We chose $600^{\circ} \mathrm{C}$ as calcination temperature as this temperature was found to have highest activity among samples calcined at different temperatures, due to the fact that the material is in the anatase phase and is highly crystalline [7]. However, there are no obvious peaks showing the presence of silver in the XRD of silver doped titania samples calcined at $600{ }^{\circ} \mathrm{C}$, which indicates that the discrete silver particles are homogeneously distributed in the titania matrix. This conclusion is further verified by studying an $\mathrm{XRD}$ of $\mathrm{Ag}-\mathrm{TiO}_{2}$ calcined at $700{ }^{\circ} \mathrm{C}$ which shows the presence of peaks corresponding to the metallic silver at 38.2, 44.4 and 64.5. Interestingly, the presence of small amounts of silver $(1,3 \mathrm{~mol} \%)$ result in the anatase to rutile phase transformation occurring at a higher temperature. This differs from other work where addition of silver resulted in the rutile phase forming at $500^{\circ} \mathrm{C}$. In samples calcinated at $700^{\circ} \mathrm{C}$, silver peaks are detected (Supplementary information, Table 1). This is also in a good agreement with the values of peaks obtaining by calcina- 

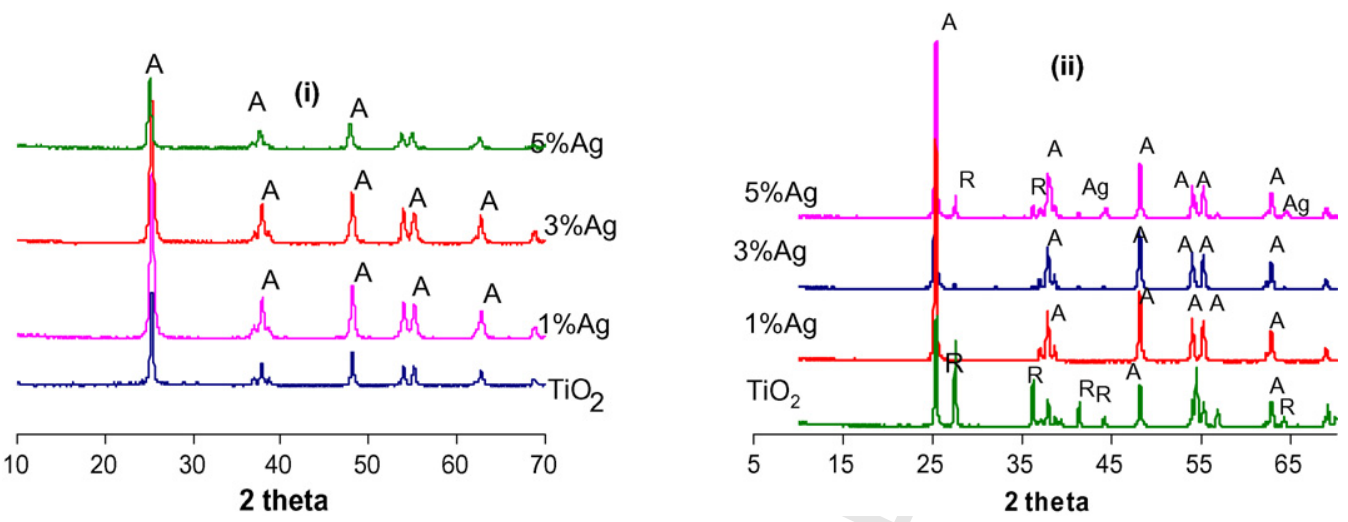

Fig. 1. XRD of: (i) undoped and silver doped titania (not pre-irradiated) all calcined at $600{ }^{\circ} \mathrm{C}$, and (ii) undoped 1,3 , and $5 \mathrm{~mol} \%$ and $\mathrm{Ag}$ doped titania all calcined at $700^{\circ} \mathrm{C}$ (peak values listed in Supplementary information, Table 1).

tion of silver nitrate alone (Supplementary information, Fig. 1a). The $700{ }^{\circ} \mathrm{C}$ sample indicates that at this higher temperature, the silver particles homogeneously distributed throughout the $\mathrm{TiO}_{2}$ matrix begin to diffuse towards the edges of the material forming agglomerates. There was no measurable difference in the XRD spectra due to pre-irradiation of samples (Fig. 2).

In order to study the effect of silver on particle size, crystallite sizes as measured from the XRD spectra (Supplementary information, Table 2). It has been found that the particle size reduces as a result of $\mathrm{Ag}$ addition. However, there is no significant reduction in particle size observed with increasing silver amounts after this initial reduction.

\subsection{Visible light absorption capability}

Diffuse reflectance spectroscopy (DRS) were used to record absorbance capacity of the powders. As a general trend, increasing amounts of $\mathrm{Ag}$ results in a higher visible absorbance capability of the materials. This is as expected, as previous workers who included silver in the titania matrix observed similar results. For example, Mills et al. used surface enhanced resonance Raman spectroscopy to investigate silver doped titania films, and observed an increased absorption in the visible region [36]. In addition, in our own laboratories we are currently

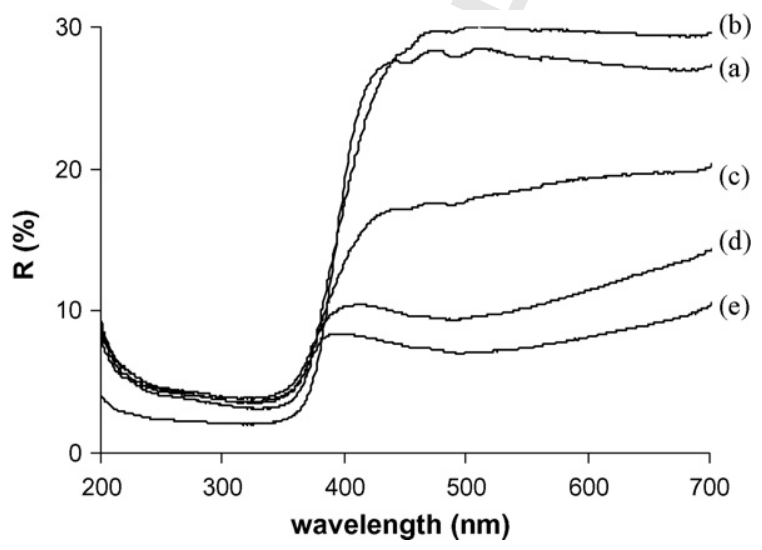

Fig. 2. Diffuse reflectance spectra of: (a) undoped, (b) $1 \mathrm{~mol} \%$, (c) $2 \mathrm{~mol} \%$ (d) $3 \mathrm{~mol} \%$ and (e) $5 \mathrm{~mol} \%$ (not pre-irradiated) silver doped titania. investigating thin films of these materials and the transmission spectra show the presence of a band at $\sim 400 \mathrm{~nm}$, which is due to the presence of silver metal. It is noted that upon illumination of $\mathrm{TiO}_{2} / \mathrm{AgNO}_{3}$ solution during the preparation time stable colloidal solutions were obtained which displays the progressive evolution of characteristic silver surface plasmon band at $400 \mathrm{~nm}$. This is indicative of the formation of nanometer sized silver metal, corresponding to a size of approximately $20 \mathrm{~nm}$ [37]. These silver metals can easily disperse into the $\mathrm{TiO}_{2}$ lattice. At this plasmon band resonance frequency, there would be efficient visible light absorption.

\subsection{Photocatalytic studies}

In order to examine the photocatalytic activity of the materials prepared we studied the effect of photodegradation of R6G in the presence of the undoped and doped materials. Silver metal can be incorporated by photoreduction of $\mathrm{Ag}^{+}[38]$ or by allowing the silver salt to decompose during calcination [39]. In order to examine the effect of pre-irradiation, we prepared two parallel sets of doped materials by the sol-gel method-the first where the silver nitrate was added and the solution irradiated for $60 \mathrm{~min}$ before drying and calcination ("pre-irradiated"), the second where the silver nitrate was added and the material dried and calcined without irradiation ("not pre-irradiated"). The results of this study are shown in Table 1. R6G showed minimal degradation in the absence of a catalyst over the same time period.

\section{Table 1}

The rate constant for degradation of R6G for samples with different mol percentages of silver-after either pre-experimental irradiation to reduce $\mathrm{AgNO}_{3}$ or sample which were directly calcinated

\begin{tabular}{lll}
\hline $\operatorname{Ag}(\mathrm{mol} \%)$ & \multicolumn{2}{l}{ Rate constant $\left(\mathrm{min}^{-1}\right)$} \\
\cline { 2 - 3 } & No pre-irradiation & Pre-irradiation $(60 \mathrm{~min})$ \\
\hline 0 & 0.06 & - \\
1 & $0.16 \pm 0.02$ & $0.10 \pm 0.01$ \\
2 & $0.18 \pm 0.02$ & $0.17 \pm 0.02$ \\
3 & $0.24 \pm 0.02$ & $0.21 \pm 0.03$ \\
5 & $0.34 \pm 0.03$ & $0.24 \pm 0.03$ \\
\hline
\end{tabular}



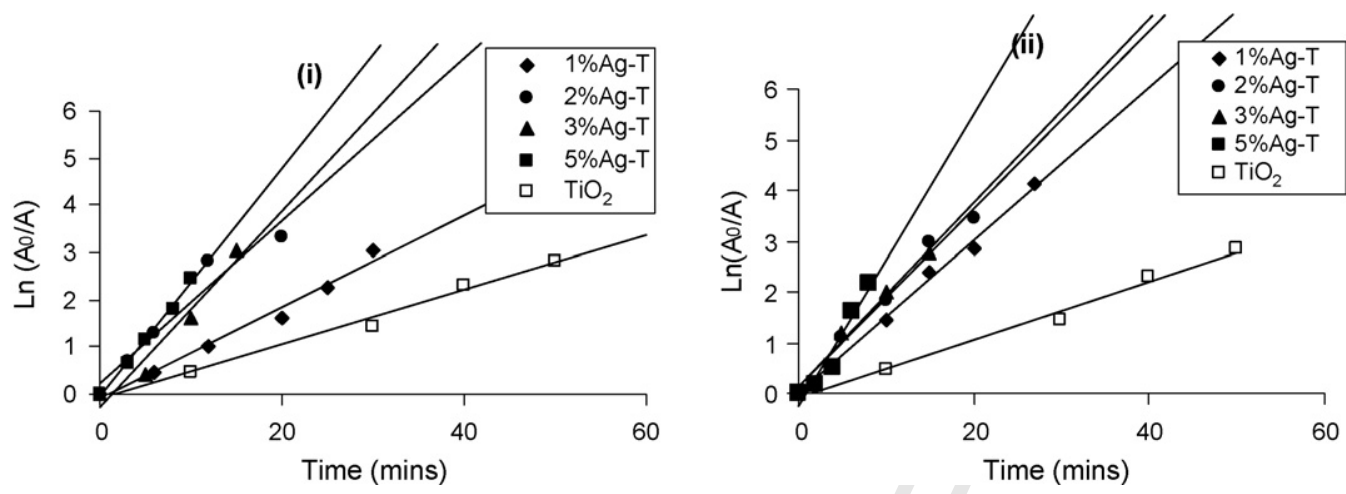

Fig. 3. Kinetic analysis showing the effect of increasing concentration of $\mathrm{Ag}$ on the rate of degradation of R6G where the preparation method involved: (i) no pre-irradiation and (ii) with pre-irradiation.
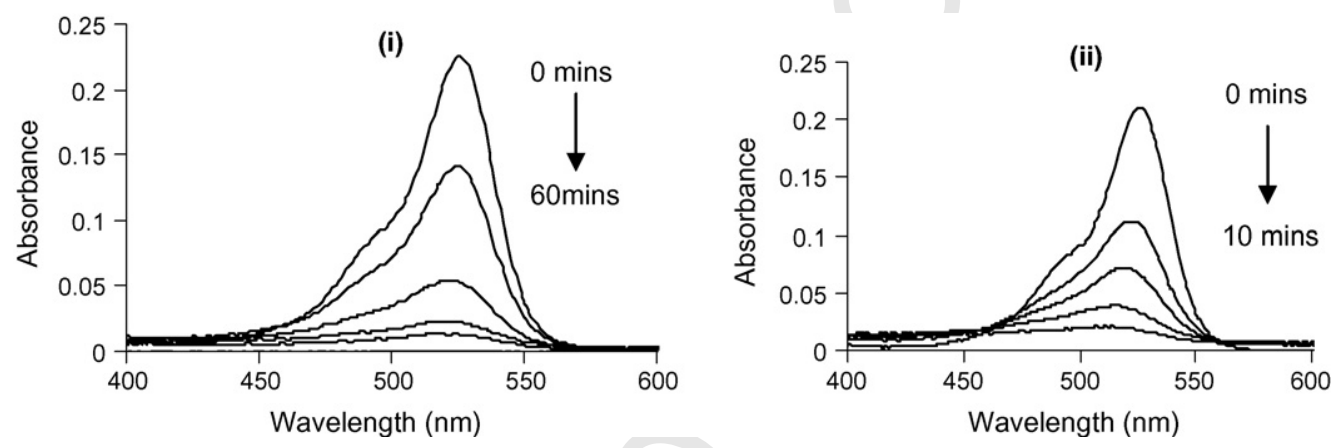

Fig. 4. Absorption spectra of the degradation of $\mathrm{R} 6 \mathrm{G}$ in presence of: (i) $\mathrm{TiO}_{2}$ and (ii) $5 \mathrm{~mol} \% \mathrm{Ag}$ doped $\mathrm{TiO}_{2}$.

For both pre-irradiated and non-irradiated samples, the activity of the catalyst is enhanced significantly on addition of silver, with the degradation rate increasing from 0.06 to $0.24 \mathrm{~min}^{-1}$ for the pre-irradiated samples and to $0.34 \mathrm{~min}^{-1}$ for the nonpre-irradiated samples, corresponding to half lives of 12.0, 2.8 and 2.4 min, respectively (Fig. 3).

When comparing the effects of pre-irradiation, the samples that were not pre-irradiated were consistently higher than those which were not pre-irradiated, although the enhancement was not marked. Sample absorption spectra for these experiments are shown in Fig. 4. A significant blue shift in the absorption maximum is observed in all silver modified materials (typically $10-12 \mathrm{~nm}$ ), but no real shift is observed for titania.

Fig. 5 shows the rate constant of degradation as a function of mol\% of silver. As observed in the transmission and DRS

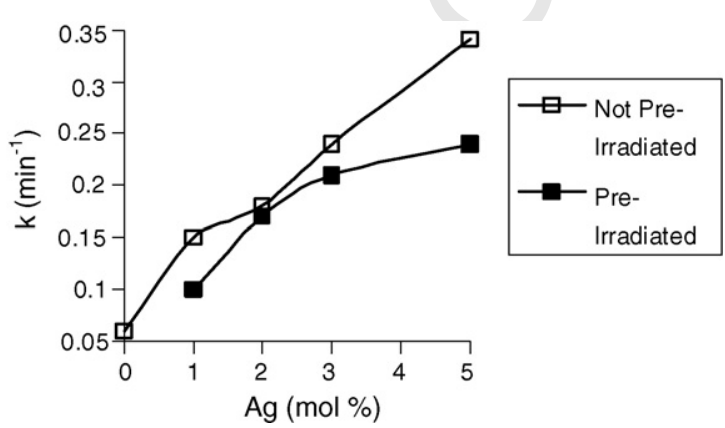

Fig. 5. Plot of rate constants of degradation against mol\% of Ag with preirradiated and non-pre-irradiated samples. spectra, increasing amounts of silver doping result in increased visible light absorption and hence enhanced photocatalytic activity. We examined the rate of degradation of $10 \mathrm{~mol} \% \mathrm{Ag}-\mathrm{TiO}_{2}$ (not pre-irradiated). A degradation rate constant of $0.07 \mathrm{~min}^{-1}$ was observed.

According to our study, it is clear that with the increase in the time of pre-irradiation, the amount of photo reduced $\mathrm{Ag}$ increases and which causes the growth of silver clusters and thereby decreasing the active sites for the photocatalytic reaction. This is confirmed by varying the pre-irradiation time of the $5 \% \mathrm{Ag}-\mathrm{TiO}_{2}$ sample, which shows the rate of degradation decrease from no pre-irradiation $\left(0.34 \mathrm{~min}^{-1}\right)$ through $20 \mathrm{~min}$ $\left(0.28 \mathrm{~min}^{-1}\right), 40 \mathrm{~min}\left(0.27 \mathrm{~min}^{-1}\right)$ and $60 \mathrm{~min}$ pre-irradiation $\left(0.24 \mathrm{~min}^{-1}\right)$.

The degradation of rhodamine $6 \mathrm{G}$ was examined in the presence of silver nanoparticles and in the absence of titania. Minimal degradation was observed over $6 \mathrm{~h}$ irradiation in the Q-Sun chamber, indicating the requirement of the presence of titania for the photocatalytic mechanism.

\subsection{Sunlight as a light source}

The above experiments were conducted in a Q-Sun solar chamber which models the solar spectrum, at a power corresponding to noon time, summer day, in Florida. We wanted to examine the ability of the materials at lower intensities. This was achieved by varying the Q-Sun irradiance intensity $(0.68$, $0.6,0.5,0.4 \mathrm{~W} / \mathrm{m}^{2}$ ) which resulted in a decrease in the efficiency of the materials (Supplementary information, Table 3). 


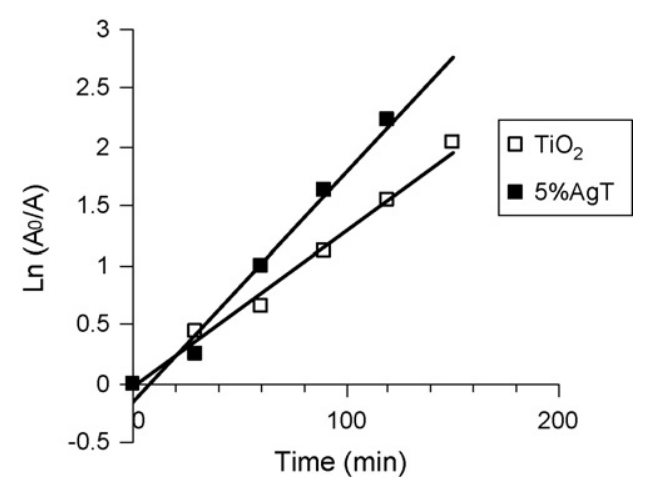

Fig. 6. Comparison of $\mathrm{TiO}_{2}$ and $5 \% \mathrm{Ag}-\mathrm{TiO}_{2}$ kinetics of degradation of $\mathrm{R} 6 \mathrm{G}$ with sunlight as a light source.

More importantly however, experiments were conducted with solutions of R6G in the presence of titania and silver doped titania $(5 \%)$ placed in a sunny window behind plate glass (cut-off $350 \mathrm{~nm}$ ). The kinetic analysis showing the rate of reaction with undoped titania and $5 \mathrm{~mol} \% \mathrm{Ag}$ doped titania (pre-irradiated) is given in Fig. 6. In this case, rate constants for decomposition are $1.95 \times 10^{-2}$ and $1.3 \times 10^{-2} \mathrm{~min}^{-1}$ for doped and undoped titania, respectively, corresponding to half lives of 52 and $35 \mathrm{~min}$. These studies show our materials to be active in maritime climates such as Ireland.

\section{Discussion}

\subsection{Characterisation of materials}

The preparation method used to synthesise titania and silver doped titania involves the use of acetic acid and titanium isopropoxide. This method has advantages over other methods in that the anatase to rutile transformation temperature is held off until approximately $700{ }^{\circ} \mathrm{C}$ [34]. Other studies in this area have reported that the anatase to rutile transformation for silver doped titania can occur at lower temperatures with increment in doping level of silver [11]. As anatase is a more photoactive form of titania, this method allows us prepare high temperature stable silver doped anatase material. XRD analysis was also performed to determine the effect of silver doping in the phase transformation at higher temperature. The titania samples prepared by this modified sol-gel method contains $48.7 \%$ rutile at $700{ }^{\circ} \mathrm{C}$ where as $5 \mathrm{~mol} \%$ silver doped sample at $700{ }^{\circ} \mathrm{C}$ contains only $13.2 \%$ rutile. Furthermore, the 1 and 3\% Ag doped titania still exist in the anatase phase at $700{ }^{\circ} \mathrm{C}$. Hence with the suitable amount of silver, the anatase to rutile transformation is pushed to an even higher temperature. This method provides for homogeneously distributed silver in samples calcined at $600{ }^{\circ} \mathrm{C}$, as the presence of silver metal is only detectable in the $5 \% \mathrm{Ag}-\mathrm{TiO}_{2}$ sample calcined at $700{ }^{\circ} \mathrm{C}$. This is also because, the $\mathrm{Ag}-\mathrm{O}$ bonding is much weaker than $\mathrm{Ti}-\mathrm{O}$ and $\mathrm{Ag}-\mathrm{Ag}$ bonding and $\mathrm{Ag}$ atom possesses higher surface free energy than the $\mathrm{TiO}_{2}$ [39]. Hence during calcination the $\mathrm{Ag}$ atoms have a tendency to aggregate into metallic Ag clusters. This is further examined by carrying out an experiment using 50:50 $\mathrm{TiO}_{2}$ :Ag mixture, calcined at $600^{\circ} \mathrm{C}$. XRD (Supplementary information, Fig. 1b) showed the presence of silver and titania peaks. It is interesting to notice that there were no silver oxide peaks or any other phases such as silver titanate present.

\subsection{Synthetic procedure and effect on photocatalytic activity}

We compared two methods for introducing metallic silver into the $\mathrm{TiO}_{2}$ material. The first was to directly reduce the $\mathrm{AgNO}_{3}$ material to $\mathrm{Ag}^{0}$ by irradiating the samples with light for a fixed time. The second was to directly calcinate the sample and allow the thermal decomposition of silver nitrate to occur according to the scheme:

$\mathrm{AgNO}_{3} \rightarrow \mathrm{Ag}_{2} \mathrm{O} \rightarrow \mathrm{Ag}$

The directly calcinated samples (i.e. non-pre-irradiated) show slightly better photocatalytic activity. It is known that silver particles deposited on the surface of the titania can act as electron-hole separation centres [40]. The radius of $\mathrm{Ag}^{+}$ions $(126 \mathrm{pm})$ is much larger than that of $\mathrm{Ti}^{4+}(68 \mathrm{pm})$ and so the $\mathrm{Ag}^{+}$ions introduced by the sol-gel process would not enter into the lattice of anatase phase. During calcination, these uniformly dispersed $\mathrm{Ag}^{+}$ions would gradually migrate from the volume of the $\mathrm{TiO}_{2}$ to the surface by enhancing their crystallinity, resulting in silver deposited on the surface on calcination [41]. Electron transfer from conduction band of $\mathrm{TiO}_{2}$ to the metallic silver particles at the interface is possible, because the Fermi level of $\mathrm{TiO}_{2}$ is higher than that of silver metal [42]. This results in the formation of Schottky barrier at the $\mathrm{Ag}-\mathrm{TiO}_{2}$ contact region, which improves the photocatalytic activity. In addition, there may be residual $\mathrm{Ag}^{+}$ions within the material, acting as efficient electron traps may contribute to the enhanced photocatalytic activity of these samples over the pre-irradiated samples. For the preirradiated samples, we propose that the main reason for reduced efficiency as compared to directly calcinated samples is that in the case of titania, photoreduced Ag cannot be highly dispersed in the surface of $\mathrm{TiO}_{2}$, so that the amount of active sites on the $\mathrm{Ag}-\mathrm{TiO}_{2}$ surface cannot markedly increase and thereby electronic structure of $\mathrm{TiO}_{2}$ cannot be changed significantly [44]. The leveling off in photocatalytic activity on further increasing amount of silver is most likely due to the fact that as the concentration of silver becomes large, it results in one of a number of effects: (i) it acts as a barrier preventing light absorption by titania, (ii) it prevents the organic substrate from contacting the titania surface and (iii) the silver may become a significant centre for electron-hole recombination [44].

It has been reported that the photodegradation of rhodamine results from both the de-ethylation and degradation of rhodamine chromophore [45]. De-ethylation is occurring on the surface and rhodamine degradation is mainly a bulk solution process. According to Amal and co-workers $[46,47]$ the enhancing effect of silver deposits on $\mathrm{TiO}_{2}$ mainly depends on the molecular structure of substrate to be oxidised. The more $\mathrm{C}=\mathrm{O}$ or $\mathrm{C}-\mathrm{O}$ bond that a molecule possess the more probable the enhancement of degradation in presence of silver. In addition, de-ethylation (a surface bound process) is characterised by a blue shift in the absorption spectrum of rhodamine on degradation [32]. In our 
samples, the silver doped titania all show significant blue shifts, whereas the titania shows little shift. This leads to the conclusion that the silver samples achieve higher surface adsorption, and hence result in better photodegradation.

These materials are visible light active. There are two parallel mechanisms which may result in electron population of the conduction band of the material-(i) that the rhodamine $6 \mathrm{G}$ absorbs visible light and injects an electron into the conduction band of the titania material, and (ii) that the material itself absorbs visible light, probably facilitated by the surface plasmon absorption of the silver doped materials. It is probable that the two mechanisms are happening in parallel, indeed some commentators have questioned the suitability of dyes such as methylene blue for photocatalytic studies [48]. However, as important as the materials ability to absorb visible light is its ability to retain charge separation, and limit recombination. In comparing our materials, we are comparing not only visible light activation, but also efficiency of the materials to subsequently form hydroxyl radicals. Therefore, photosensitisation, if it is occurring, would enhance both titania samples and silver modified samples. The role of silver is clearly limiting the recombination rate and subsequently allowing a greater proportion of hydroxyl radicals to form.

\section{Conclusions}

This paper describes the synthesis of $\mathrm{TiO}_{2}$ containing different amounts of silver metal by the sol-gel route. The synthetic method where the silver nitrate was not reduced by light before calcination resulted in a slightly more efficient photocatalysis over that which did. All materials with added silver showed visible light activation in both the Q-Sun solar simulator and Dublin sunlight, with the activity increasing with increasing amounts of silver.

\section{Uncited references}

$$
[27,30,43]
$$

\section{Acknowledgement}

RG would like to thank the Postgraduate R\&D Skills Programme (Strand 1) for funding for this work.

\section{Appendix A. Supplementary data}

Supplementary data associated with this article can be found, in the online version, at doi:10.1016/j.jphotochem.2007.02.010.

\section{References}

[1] A. Fujishima, T.N. Rao, D.A. Tryk, J. Photochem. Photobiol. C 1 (2000) 1.

[2] M.R. Hoffmann, S.T. Martin, W. Choi, D.W. Bahnemann, Chem. Rev. 95 (1995) 69.

[3] A. Fujishima, K. Honda, Nature 238 (1972) 37.

[4] T. Hisanga, K. Harada, K. Tanaka, J. Photochem. Photobiol. A 54 (1990) 113.
[5] K.N.P. Kumar, K. Keizer, A.J. Burggraaf, T. Okubo, Nature 358 (1992) 48.

[6] C. Wang, J. Ying, Chem. Mater. 11 (1999) 3113.

[7] S.C. Pillai, P. Periyat, R. George, D.E. McCormack, M.K. Seery, H. Hayden, J. Colreavy, D. Corr, S.J. Hinder, J. Phys. Chem. C 111 (4) (2007) $1605-1611$

[8] Y.C. Yeh, T.T. Tseng, D.A. Chang, J. Am. Ceram. Soc. 72 (1989) 1472.

[9] P.S. Awati, S.V. Awate, P.P. Shah, V. Ramaswamy, Catal. Commun. 4 (2003) 393.

[10] Y.H. Hsien, C.F. Chang, Y.H. Chen, S. Chen, Appl. Catal. B 31 (2001) 241.

[11] H.E. Chao, Y.U. Yun, H.U. Xiangfang, A. Larbot, J. Eur. Ceram. Soc. 23 (2003) 1457.

[12] D. Chatterjee, S. Dasguptha, J. Photochem. Photobiol. C 6 (2005) 186.

[13] Y. Ichihashi, H. Yamashita, M. Anpo, Funct. Mater. 16 (1996) 12.

[14] E. Hendry, F. Wang, J. Shan, T.F. Heins, M. Bonn, Phys. Rev. B 69 (2004) 81.

[15] G. Zhao, H. Kozuka, T. Yoko, Thin Solid Films 277 (1996) 147.

[16] S. Banerjee, J. Gopal, P. Muraleedharan, A.K. Tyagi, B. Raj, Curr. Sci. 90 (2006) 10.

[17] W. Choi, A. Termin, M.R. Hoffmann, J. Phys. Chem. 98 (1994) 13669.

[18] P.D. Cozzoli, R. Comparelli, E. Fanizza, M.L. Curri, A. Agostiano, D. Laub, J. Am. Chem. Soc. 126 (2004) 3868.

[19] K.S. Mayya, D.I. Gittins, F. Caruso, Chem. Mater. 13 (2001) 3833.

[20] M. Machida, K. Norimoto, T. Kimura, J. Am. Ceram. Soc. 88 (2005) 95Y.

[21] I. Ilisz, A. Dombi, Appl. Catal. A 180 (1999) 35.

[22] E. Stathatos, T. Petrova, P. Lianos, Langmuir 17 (2001) 5025

[23] P.V. Kamat, J. Phys. Chem. B 106 (2002) 7729.

[24] M. Jacob, H. Levanon, P.V. Kamat, Nanoletters 3 (2003) 353.

[25] E. Bae, W. Choi, Environ. Sci. Technol. 37 (2003) 147.

[26] J.M. Hermann, H. Tahiri, Y. Ait-Ichou, G. Lossaletta, A.R. Gonzalez-Elipe, A. Fernandez, Appl. Catal. B 13 (1997) 219.

[27] D. Bahnemann, Solar Energy 77 (2004) 445

[28] A. World, Chem. Mater. 5 (1993) 280.

[29] V. Vamathevan, R. Amal, D. Beydoun, G. Low, S. McEvoy, J. Photochem. Photobiol. A 148 (2002) 233.

[30] P. Falaras, I.M. Arabatzis, T. Stergioulos, M.C. Bernard, Int. J. Photoenergy 5 (2003) 123.

[31] M. Sokmen, D.W. Allen, F. Akkas, N. Kartel, F. Acar, Water, Air Soil Pollut. 132 (2001) 153.

[32] H.M. Sung-suh, J.R. Choi, H.J. Hah, S.M. Koo, Y.C. Bae, J. Photochem. Photobiol. A 163 (2004) 37.

[33] L. Zhang, J.C. Yu, H.Y. Yip, Q. Li, K.W. Kwong, A. Xu, P.K. Wong, Langmuir 19 (2003) 10372.

[34] C. Suresh, V. Biju, P. Mukundan, K.G.K. Warrier, Polyhedron 17 (1998) 3131.

[35] http://www.solarlight.com/products/PMA2107.html (accessed November 2006).

[36] A. Mills, G. Hill, M. Stewart, D. Graham, W.E. Smith, S. Hodgen, P.J. Halfpenny, K. Faulds, P. Robertson, Appl. Spectrosc. 58 (2004) 922

[37] Y. Wang, N. Herron, J. Phys. Chem. 95 (1991) 525.

[38] C. Hu, Y.Q. Lan, J.H. Qu, X.X. Hu, A.M.D. Wang, J. Phys. Chem. B 110 (2006) 4066.

[39] A. Chen, M.C. Bartelt, S.M. Seutter, K.F. McCarty, Surf. Sci. 464 (2000) L708.

[40] A. Henglein, J. Phys. Chem. 83 (1979) 2209.

[41] W.D. Kingery, H.K. Bowen, D.R. Uhlmann, Introduction to Ceramics, second ed., Wiley, New York, 1976, p. 457.

[42] A. Sclafani, J.M. Hermann, J. Photochem. Photobiol. A 113 (1998) 181.

[43] V. Iliev, D. Tomova, L. Bilyarska, A. Eliyas, L. Petrov, Appl. Catal. B 63 (2006) 266.

[44] B. Xin, L. Jing, Z. Ren, B. Wang, H. Fu, J. Phys. Chem. B 109 (2005) 2805.

[45] T. Watanabe, T. Takizava, K. Honda, J. Phys. Chem. 81 (1977) 1845.

[46] H. Tran, K. Chiang, J. Scott, R. Amal, Photochem. Photobiol. Sci. 4 (2005) 565.

[47] H. Tran, J. Scott, K. Chiang, R. Amal, J. Photochem. Photobiol. A 183 (2006) 41.

[48] X. Yan, T. Ohno, K. Nishijima, R. Abe, B. Ohtani, Chem. Phys. Lett. 429 (2006) 606. 